\title{
Prognostic Value of the Number of Removed Lymph Nodes in Vulvar Squamous Cell Carcinoma Patients With Node-Positive Disease: A Population-Based Study
}

\author{
San-Gang Wu't, Wen-Wen Zhang ${ }^{2 t}$, Jia-Yuan Sun', Qiong-Hua Chen ${ }^{3}$, Zhen-Yu He ${ }^{2 *}$ \\ and Juan Zhou ${ }^{3 *}$ \\ ${ }^{1}$ Department of Radiation Oncology, Xiamen Cancer Hospital, The First Affiliated Hospital of Xiamen University, Xiamen, \\ China, ${ }^{2}$ Department of Radiation Oncology, Sun Yat-sen University Cancer Center, State Key Laboratory of Oncology in \\ South China, Collaborative Innovation Center of Cancer Medicine, Guangzhou, China, ${ }^{3}$ Department of Obstetrics and \\ Gynecology, The First Affiliated Hospital of Xiamen University, Xiamen, China
}

\section{OPEN ACCESS}

Edited by:

Alice de Medeiros Zelmanowicz, Federal University of Health Sciences

of Porto Alegre, Brazil

Reviewed by:

Mila Pontremoli Salcedo,

Federal University of Health Sciences

of Porto Alegre, Brazil

Luigi Formisano,

Vanderbilt University Medical Center,

United States

*Correspondence:

Zhen-Yu He

hezhy@sysucc.org.cn;

Juan Zhou

juanzhou12345@163.com

these authors have contributed equally to this work.

Specialty section:

This article was submitted

to Women's Cancer,

a section of the journal

Frontiers in Oncology

Received: 16 January 2018

Accepted: 09 May 2018

Published: 30 May 2018

Citation:

Wu S-G, Zhang W-W, Sun J-Y,

Chen Q-H, He Z-Y and Zhou J (2018)

Prognostic Value of the Number of

Removed Lymph Nodes in Vulvar

Squamous Cell Carcinoma Patients

With Node-Positive Disease:

A Population-Based Study.

Front. Oncol. 8:184.

doi: 10.3389/fonc.2018.00184
Introduction: To investigate the effect of the number of removed lymph nodes (RLNs) on outcomes in patients with node-positive vulvar squamous cell carcinoma (SCC).

Methods: This population-based retrospective study included vulvar SCC patients recorded on the surveillance, epidemiology, and end results database, who received surgery and lymphadenectomy. Cox regression proportional hazards were used for multivariate analysis. The number of RLNs was examined as a 4-level categorical variable based on quartiles.

Results: In total, 703 patients were identified. Patients with a higher RLN count had a significantly higher number of positive lymph nodes. The 3-year cause-specific survival (CSS) rates were 48.9, 65.9, 73.1, and 67.3\% in patients with 1-6, 7-10, 11-16, and $17-45$ RLNs, respectively ( $p<0.001$ ), and the 3-year overall survival (OS) rates were $36.1,50.6,61.1$, and $57.6 \%$, for the same RLN groups, respectively ( $p<0.001$ ). RLN count was an independent predictor of outcome. Using 7-10 RLNs as reference, patients with 1-6 RLNs had poor CSS [hazard ratio $(\mathrm{HR}) 1.727$, 95\% confidence interval $(\mathrm{Cl})$ $1.201-2.485, p=0.003$ ] and OS (HR 1.436, 95\% Cl 1.078-1.911, $p=0.013$ ), while there were comparable outcomes in patients with 11-16 and 17-45 RLNs to patients with 7-10 RLNs. Adjuvant radiotherapy improved CSS ( $p=0.023)$ and OS ( $p=0.003)$ in patients with $\leq 6 \mathrm{RLNs}$, but was not associated with better outcomes in patients with $>6$ RLNs.

Conclusion: The removal of more than six lymph nodes improves vulvar SCC outcomes in patients with node-positive disease.

Keywords: vulvar cancer, lymphadenectomy, removed node count, survival, surveillance, epidemiology, and end result

\section{INTRODUCTION}

Vulvar squamous cell carcinoma (SCC) is a rare gynecological malignancy, accounting for approximately $3-5 \%$ of all gynecological cancers $(1,2)$. Lymph node status is an important indicator for predicting the outcome of vulvar SCC. One meta-analysis showed the 5-year overall survival (OS) rate to be $84.5,58.5,47.4$, and $30.1 \%$ in patients with node-negative disease, one positive lymph 
node (PLN), two PLNs, and more than three PLNs, respectively (3). The standard treatment procedure for patients with nodepositive vulvar SCC is radical inguinal-femoral lymphadenectomy. However, controversy exists regarding the optimal extent of inguinal-femoral lymphadenectomy in patients with vulvar cancer.

Theoretically, removing more lymph nodes reduces the potential for lymph node micrometastases, reducing the risk of relapse and improving survival. Several previous studies have found that in patients with node-negative vulvar SCC, an increased number of removed lymph nodes (RLNs) was significantly associated with better outcomes (4-8). In patients with early-stage vulvar SCC, sentinel lymph node biopsy (SLNB) has been confirmed as safe and feasible (9-11), with no significant difference in clinical outcomes between inguinal-femoral lymphadenectomy and SLNB (12). Therefore, inguinal-femoral lymphadenectomy may be avoided in patients with early-stage vulvar SCC after careful lymph node assessment. A study of a population-based cohort from the surveillance, epidemiology, and end results (SEER) program has confirmed that a higher RLN count found during lymphadenectomy was associated with significantly better disease-specific survival in patients with stage III node-negative vulvar SCC, but not in patients with early-stage vulvar cancer (6).

The number of PLNs is significantly related to survival outcomes in patients with vulvar SCC; therefore, the extent of lymphadenectomy could be a more accurate assessment of lymph node status of patients. However, the optimal extent of lymphadenectomy in patients with node-positive disease remains controversial $(13,14)$. A population-based study to investigate the role of the RLN count in vulvar SCC is important, due to the rarity of the disease. The purpose of this retrospective population-based study was to assess the effect of RLN count on outcome in patients with node-positive vulvar SCC treated with lymphadenectomy.

\section{MATERIALS AND METHODS}

\section{Patients}

This population-based study identified vulvar cancer patients diagnosed between 2004 and 2013, using the SEER program. This program, maintained by the National Cancer Institute, covers approximately $28 \%$ of the United States population and includes information on the demographics, incidence, and outcomes of specific cancers (15). We have obtained the permission to access the publicly SEER database with the reference number 11025-Nov 2016. We included patients who met the following criteria: (1) histologically confirmed node-positive vulvar SCC who received surgery including lymphadenectomy; (2) the exact number of RLNs and PLNs were recorded; and (3) patient characteristics including age, race/ethnicity, tumor grade, tumor size, and receipt of radiotherapy or chemotherapy were available. Patients who received preoperative radiotherapy or SLNB were excluded. The institutional review board of the First Affiliated Hospital of Xiamen University had approved this study.

The following demographic, clinicopathological, and treatment characteristics were included: age, race/ethnicity, tumor grade, tumor size, receipt of radiotherapy or chemotherapy, and the number of PLNs and RLNs. The primary endpoints were cause-specific survival (CSS) and OS. CSS was defined as time from initial diagnosis to the date of vulvar cancer-related death. OS was defined as time from initial diagnosis to the date of death or last follow-up.

\section{Statistical Analysis}

The $\chi^{2}$ test, Fisher's exact test, and one-way analysis of variance were used to compare the differences in patient characteristics among the RLN groups. Recognizing that the number of RLNs may have been incompletely counted or that there may be natural interindividual variation in lymph node distribution, the variable was examined as a 4-level categorical variable based on quartiles. Survival curves were plotted using the Kaplan-Meier method and compared using the log-rank test. Cox regression analysis was used to identify significant prognostic factors. Variables with $p<0.05$ in the univariate analysis were entered into multivariate Cox regression models. All statistical tests were conducted using SPSS version 22 statistical software (IBM Corporation, Armonk, NY, USA). A $p$ value less than 0.05 was considered statistically significant.

\section{RESULTS}

\section{Patients Characteristics and Number of RLNs}

A total of 703 patients were included and the patient characteristics are displayed in Table 1. The median age of the patients was 69 years (range $21-95$ years). Of the patients, $79.1 \%(n=556)$ were non-Hispanic White. The median tumor size was $35 \mathrm{~mm}$ (range 2-200 mm). A total of $490(69.7 \%)$ and $204(29.0 \%)$ patients were received adjuvant radiotherapy and chemotherapy, respectively.

The median RLN count was 12 (25th percentile: 6 , 75th percentile: 16; range 1-45) and the median PLN count was 2 (range 1-17). Figure 1 shows the distribution of number of RLNs. The number of RLNs was classified using quartiles as follows: Group $1(1-6, n=181)$, Group $2(7-10, n=171)$, Group 3 (11-16, $n=194)$, and Group $4(17-45, n=157)$. Patients with a higher RLN count were more likely to be elderly $(p=0.008)$. In addition, a higher PLN count was seen in patients with a higher RLN count, and Group 2, 3, and 4 patients had a significantly higher number of RLNs compared with Group 1. There were no associations between race/ethnicity, grade, tumor size, and receipt of adjuvant therapy among the PLN groups.

\section{Correlating the Number of PLNs With Survival}

The median follow-up was 21.0 months (range 0-119 months). A total of 344 patients died and 230 (66.9\%) of these died from vulvar cancer-related diseases. The 3 -year CSS and OS rates were 64.1 and $51.5 \%$, respectively.

A higher RLN count was associated with better CSS and OS. In the four categories of RLNs, the 3-year CSS rates were 48.9, 65.9, 73.1 , and $67.3 \%$ for patients in Groups 1, 2, 3, and 4, respectively $(p<0.001)$ (Figure 2A). The 3-year OS rates were 36.1, 50.6, 
TABLE 1 | The baseline characteristics of 703 vulvar cancer patients.

\begin{tabular}{|c|c|c|c|c|c|c|}
\hline \multirow[t]{2}{*}{ Variable } & \multirow[b]{2}{*}{ All } & \multicolumn{5}{|c|}{ Number of RLNs } \\
\hline & & $1-6$ & $7-10$ & $11-16$ & $17-45$ & $p$-Value \\
\hline Age (years) (mean $\pm \mathrm{SD}$ ) & $67.0 \pm 14.3$ & $69.7 \pm 14.7$ & $67.4 \pm 15.2$ & $64.8 \pm 13.8$ & $66.1 \pm 13.2$ & 0.008 \\
\hline \multicolumn{7}{|l|}{ Race } \\
\hline Non-Hispanic White & 556 & $139(76.8)$ & $137(80.1)$ & $156(80.4)$ & $124(79.0)$ & 0.966 \\
\hline Non-Hispanic Black & 49 & $15(8.3)$ & $10(5.8)$ & $14(7.2)$ & $10(6.4)$ & \\
\hline Hispanic (all Races) & 76 & $21(11.6)$ & $20(11.7)$ & $19(9.8)$ & $16(10.2)$ & \\
\hline Other & 22 & $6(3.3)$ & $4(2.3)$ & $5(2.6)$ & $7(4.5)$ & \\
\hline \multicolumn{7}{|l|}{ Tumor grade } \\
\hline Well differentiated & 98 & $30(16.6)$ & $24(14.0)$ & $21(10.8)$ & $23(14.6)$ & 0.534 \\
\hline Moderately differentiated & 369 & $96(53.0)$ & $82(48.0)$ & $109(56.2)$ & $82(52.2)$ & \\
\hline Poorly/undifferentiated & 236 & $55(30.4)$ & $65(38.0)$ & $64(33.0)$ & $52(33.1)$ & \\
\hline Tumor size $(\mathrm{mm})($ mean $\pm \mathrm{SD})$ & $39.4 \pm 23.2$ & $40.1 \pm 20.6$ & $39.6 \pm 27.1$ & $38.0 \pm 23.2$ & $40.0 \pm 21.6$ & 0.819 \\
\hline \multicolumn{7}{|l|}{ Number of PLNs (n) } \\
\hline Mean \pm SD & $2.3 \pm 2.1$ & $1.7 \pm 0.9$ & $2.4 \pm 2.0$ & $2.5 \pm 2.0$ & $2.9 \pm 2.8$ & $<0.001$ \\
\hline 1 & 320 & $98(54.1)$ & $85(49.7)$ & $78(40.2)$ & $59(37.6)$ & $<0.001$ \\
\hline 2 & 174 & $53(29.3)$ & 33 (19.3) & $52(26.8)$ & $36(22.9)$ & \\
\hline$\geq 3$ & 209 & $30(16.6)$ & $53(31.0)$ & $64(33.0)$ & $62(39.5)$ & \\
\hline \multicolumn{7}{|l|}{ Adjuvant radiotherapy } \\
\hline No & 213 & $62(34.3)$ & $55(32.2)$ & $48(24.7)$ & $48(30.6)$ & 0.215 \\
\hline Yes & 490 & $119(65.7)$ & $116(67.8)$ & $146(75.3)$ & $109(69.4)$ & \\
\hline \multicolumn{7}{|l|}{ Chemotherapy } \\
\hline No/unknown & 499 & $127(70.2)$ & 117 (68.4) & $144(74.2)$ & $111(70.7)$ & 0.659 \\
\hline Yes & 204 & $54(29.8)$ & 54 (61.6) & $50(25.8)$ & 46 (29.3) & \\
\hline
\end{tabular}

PLN, positive lymph node; RLN, removed lymph node.

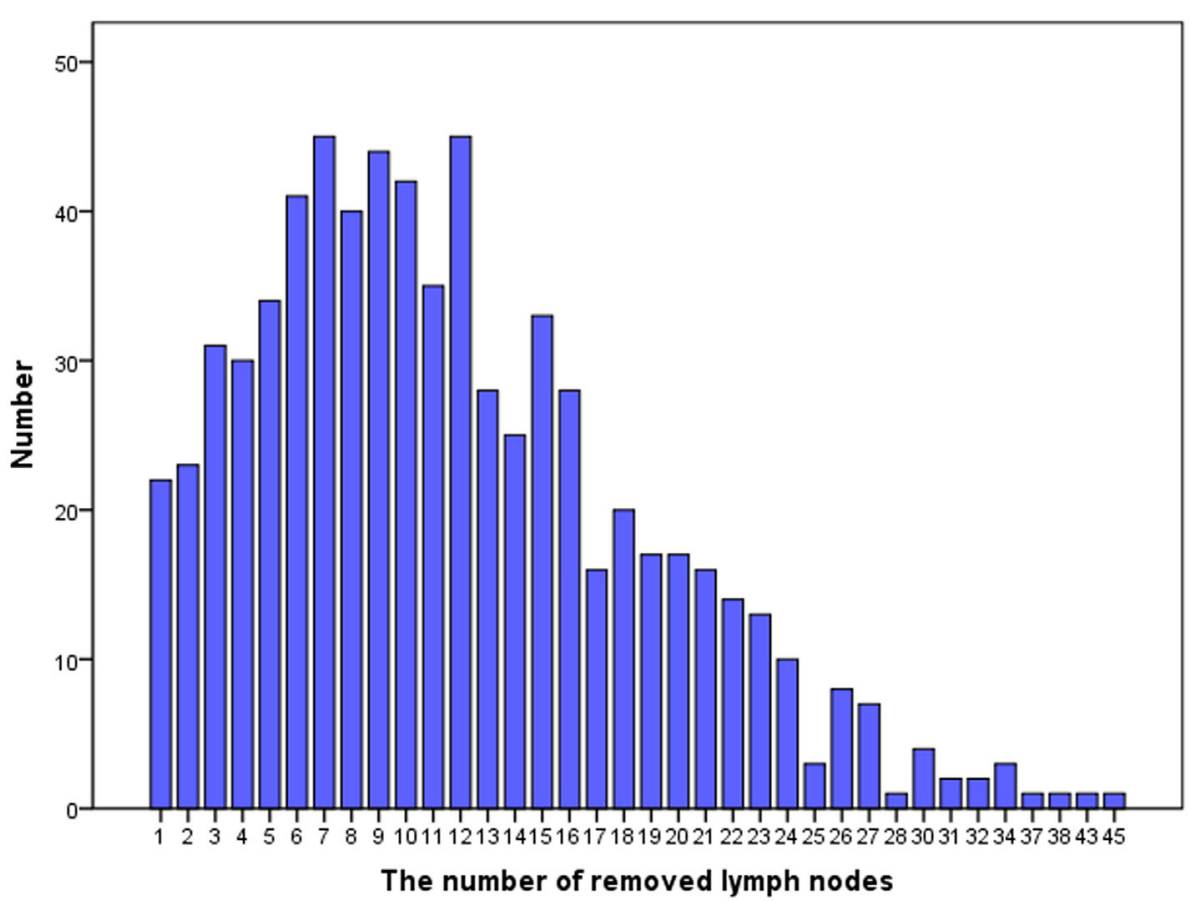

FIGURE 1 | Distribution of number of resected lymph nodes.

61.1 , and $57.6 \%$ for patients in Groups 1, 2, 3, and 4, respectively $(p<0.001)$ (Figure 2B). However, there was no significant difference in CSS $(p=0.409)$ and OS $(p=0.079)$ among Groups 2 , 3 , and 4 .

\section{Analysis of Prognostic Factors on Outcomes}

The results of Cox regression univariate analyses indicated that age, race/ethnicity, tumor size (continuous variable), PLN count, 

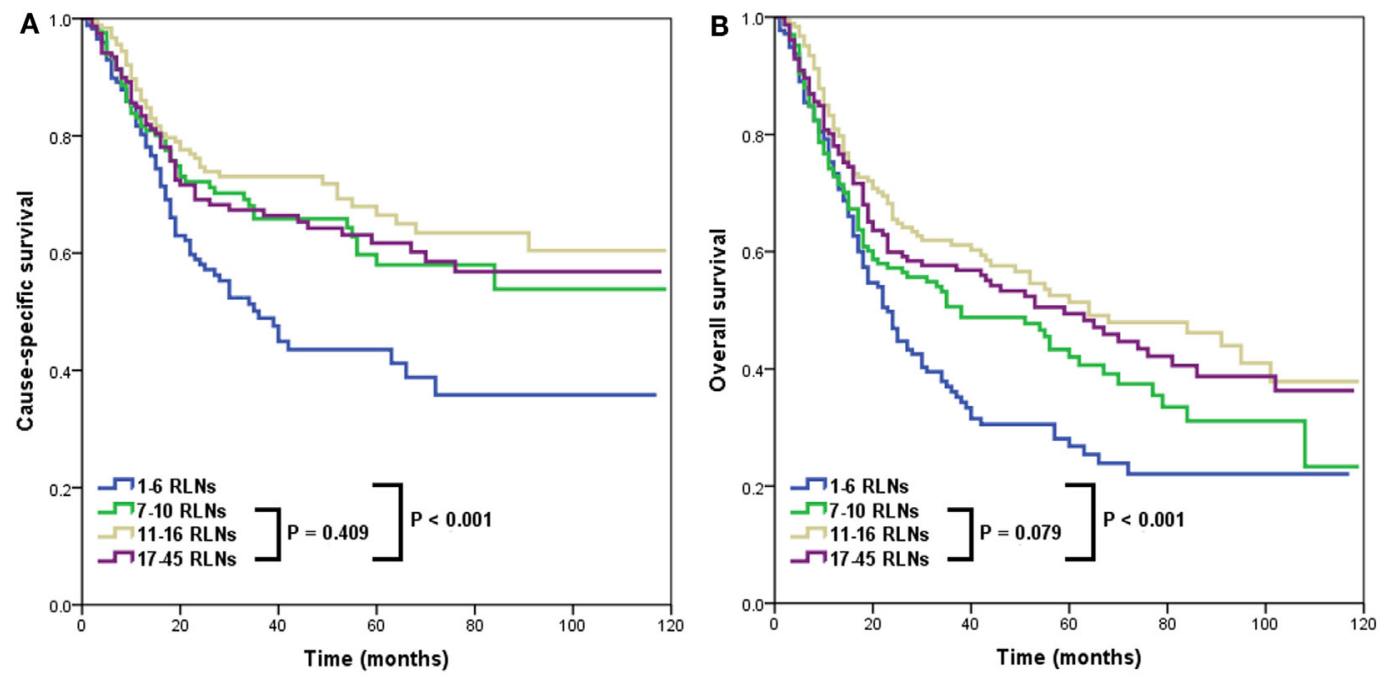

FIGURE 2 | Impact of the number of removed lymph nodes (RLNs) on cause-specific survival (A) and overall survival (B).

TABLE 2 | Univariate Cox regression analysis of prognostic factors of 703 vulvar cancer patients.

\begin{tabular}{|c|c|c|c|c|c|c|}
\hline \multirow[t]{2}{*}{ Variables } & \multicolumn{3}{|c|}{ css } & \multicolumn{3}{|c|}{ os } \\
\hline & HR & $95 \% \mathrm{Cl}$ & $p$-Value & HR & $95 \% \mathrm{Cl}$ & $p$-Value \\
\hline Age (continuous variable) & 1.037 & $1.027-1.048$ & $<0.001$ & 1.041 & $1.032-1.049$ & $<0.001$ \\
\hline \multicolumn{7}{|l|}{ Race } \\
\hline Non-Hispanic White & 1 & & & 1 & & \\
\hline Non-Hispanic Black & 0.478 & $0.245-0.932$ & 0.030 & 0.698 & $0.444-1.098$ & 0.119 \\
\hline Hispanic (all Races) & 0.881 & $0.581-1.336$ & 0.550 & 0.898 & $0.643-1.255$ & 0.529 \\
\hline Other & 0.312 & $0.100-0.977$ & 0.046 & 0.403 & $0.179-0.904$ & 0.027 \\
\hline \multicolumn{7}{|l|}{ Tumor grade } \\
\hline Well differentiated & 1 & & & 1 & & \\
\hline Moderately differentiated & 0.937 & $0.646-1.358$ & 0.730 & 0.972 & $0.719-1.314$ & 0.853 \\
\hline Poorly/undifferentiated & 0.852 & $0.570-1.272$ & 0.433 & 0.879 & $0.635-1.217$ & 0.436 \\
\hline Tumor size (continuous variable) & 1.013 & $1.009-1.018$ & $<0.001$ & 1.012 & $1.008-1.016$ & $<0.001$ \\
\hline \multicolumn{7}{|l|}{ Number of PLNs (n) } \\
\hline 1 & 1 & & & 1 & & \\
\hline 2 & 1.408 & $1.007-1.969$ & 0.046 & 1.156 & $0.879-1.521$ & 0.3 \\
\hline$\geq 3$ & 2.367 & $1.753-3.197$ & $<0.001$ & 2.208 & $1.744-2.796$ & $<0.001$ \\
\hline \multicolumn{7}{|l|}{ Number of RLNs $(n)$} \\
\hline $7-10$ & 1 & & & 1 & & \\
\hline $1-6$ & 1.588 & $1.113-2.266$ & 0.011 & 1.335 & $1.008-1.767$ & 0.044 \\
\hline $11-16$ & 0.782 & $0.530-1.153$ & 0.215 & 0.712 & $0.527-0.961$ & 0.027 \\
\hline $17-45$ & 0.962 & $0.653-1.416$ & 0.844 & 0.817 & $0.603-1.107$ & 0.192 \\
\hline \multicolumn{7}{|l|}{ Adjuvant radiotherapy } \\
\hline No & 1 & & & 1 & & \\
\hline Yes & 0.806 & $0.612-1.062$ & 0.126 & 0.721 & $0.580-0.895$ & 0.003 \\
\hline \multicolumn{7}{|l|}{ Chemotherapy } \\
\hline No/unknown & 1 & & & 1 & & \\
\hline Yes & 0.917 & $0.688-1.222$ & 0.556 & 0.744 & $0.584-0.947$ & 0.016 \\
\hline
\end{tabular}

Cl, confidence interval; CSS, cause-specific survival; HR, hazard ratio; OS, overall survival; PLN, positive lymph node; RLN, removed lymph node.

and RLN count were prognostic factors in CSS and OS. In addition, receipt of radiotherapy or chemotherapy was associated with better CSS and OS (Table 2).

The results of multivariate Cox regression analyses showed that RLN count was an independent predictor for outcomes, with a higher RLN count associated with better CSS and OS. Using an RLN count of 7-10 as reference, patients with 1-6 RLNs had poor CSS [hazard ratio (HR): $1.727,95 \%$ confidence interval (CI): 1.201-2.485, $p=0.003$ ] and OS (HR: 1.436, 95\% CI: 1.078-1.911, $p=0.013)$, while there were comparable outcomes in patients 
with 11-16 RLNs and 17-45 RLNs compared with patients with 7-10 RLNs. Age, race/ethnicity, tumor size, and the number of PLNs were also the independent predictors for survival outcomes (Table 3).

\section{Effect of Adjuvant Radiotherapy on Outcomes According to Number of RLNs}

Adjuvant radiotherapy was not an independent predictor in our multivariate analyses. However, several studies have indicated that adjuvant radiotherapy is associated with an improvement of outcomes in patients with node-positive vulvar SCC (16-19). Therefore, we further analyzed the effect of adjuvant radiotherapy on survival outcomes according to the number of RLNs. Our results showed that in patients with $\leq 6$ RLNs, adjuvant radiotherapy was associated with better CSS $(p=0.023)$ (Figure 3A) and $\mathrm{OS}(p=0.003)$ (Figure 3B), while adjuvant radiotherapy was not associated with improved CSS $(p=0.740)$ or OS $(p=0.097)$ in patients with $>6$ RLNs.

TABLE 3 | Multivariate Cox regression analysis of prognostic factors of 703 vulvar cancer patients.

\begin{tabular}{|c|c|c|c|c|c|c|}
\hline \multirow[t]{2}{*}{ Variables } & \multicolumn{3}{|c|}{ CSS } & \multicolumn{3}{|c|}{ os } \\
\hline & HR & $95 \% \mathrm{Cl}$ & $p$-Value & HR & $95 \% \mathrm{Cl}$ & $p$-Value \\
\hline Age (continuous variable) & 1.033 & $1.023-1.044$ & $<0.001$ & 1.039 & $1.031-1.048$ & $<0.001$ \\
\hline \multicolumn{7}{|l|}{ Race } \\
\hline Non-Hispanic White & 1 & & & 1 & & \\
\hline Non-Hispanic Black & 0.625 & $0.317-1.233$ & 0.175 & 0.972 & $0.610-1.547$ & 0.904 \\
\hline Hispanic (all Races) & 0.888 & $0.581-1.358$ & 0.583 & 0.930 & $0.663-1.306$ & 0.677 \\
\hline Other & 0.312 & $0.099-0.980$ & 0.046 & 0.410 & $0.182-0.925$ & 0.032 \\
\hline Tumor size (continuous variable) & 1.012 & $1.007-1.017$ & $<0.001$ & 1.011 & $1.007-1.015$ & $<0.001$ \\
\hline \multicolumn{7}{|l|}{ Number of PLNs $(n)$} \\
\hline 1 & 1 & & & 1 & & \\
\hline 2 & 1.280 & $0.912-1.797$ & 0.153 & 1.045 & $0.792-1.379$ & 0.756 \\
\hline$\geq 3$ & 2.390 & $1.751-3.264$ & $<0.001$ & 2.166 & $1.696-2.764$ & $<0.001$ \\
\hline \multicolumn{7}{|l|}{ Number of RLNs (n) } \\
\hline $7-10$ & 1 & & & 1 & & \\
\hline $1-6$ & 1.727 & $1.201-2.485$ & 0.003 & 1.436 & $1.078-1.911$ & 0.013 \\
\hline $11-16$ & 0.864 & $0.583-1.281$ & 0.468 & 0.809 & $0.597-1.096$ & 0.171 \\
\hline $17-45$ & 1.023 & $0.692-1.514$ & 0.908 & 0.871 & $0.641-1.183$ & 0.376 \\
\hline
\end{tabular}

\section{Adjuvant radiotherapy}

No

Yes

-

0.982

$0.774-1.247$

\section{Chemotherapy}

No/unknown

Yes

1

Cl, confidence interval; CSS, cause-specific survival; HR, hazard ratio; OS, overall survival; PLN, positive lymph node; RLN, removed lymph node.
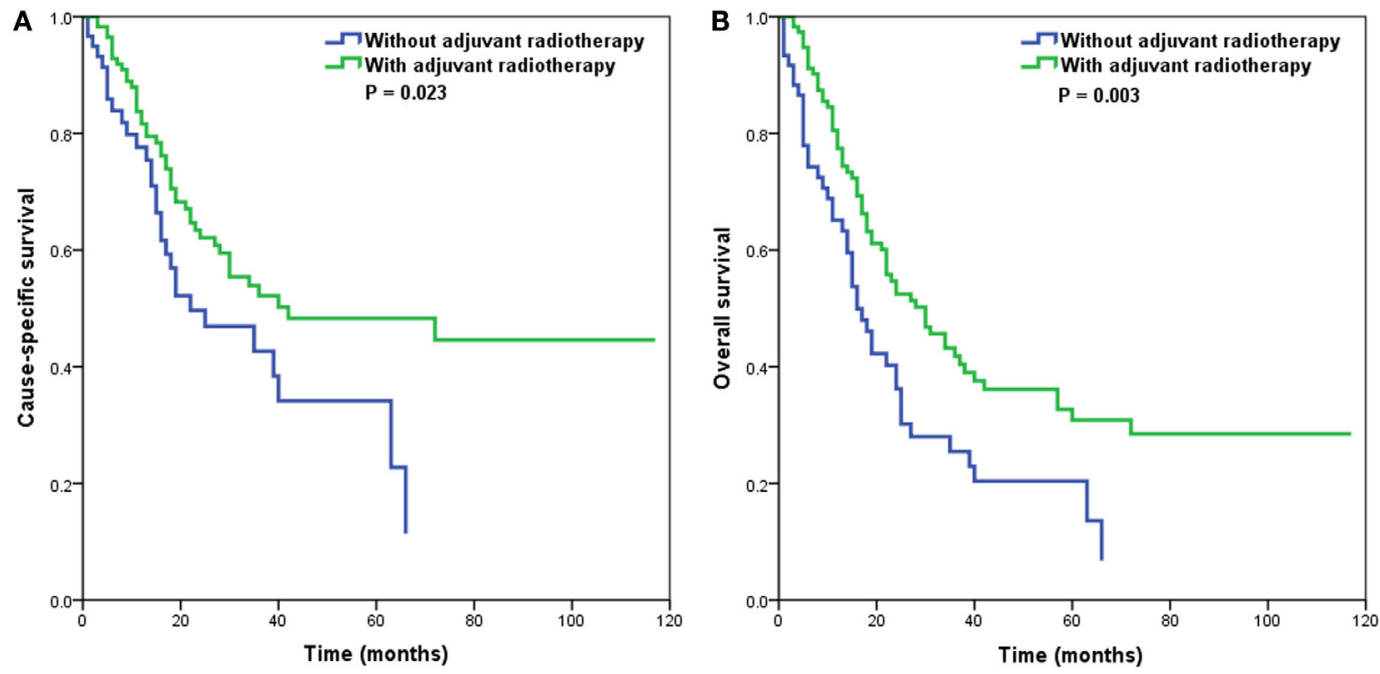

FIGURE 3 | Impact of adjuvant radiotherapy on cause-specific survival (A) and overall survival (B) in patients with 1-6 removed lymph nodes. 


\section{DISCUSSION}

In this retrospective study, we used a population-based cohort to investigate clinical outcomes according to number of RLNs in patients with node-positive vulvar SCC, and our results indicated that patients with $\leq 6$ RLNs had a higher risk of death compared with patients with $>6$ RLNs.

Lymph node status has been confirmed as an important indicator in prognostic assessment, and has been included in the staging system for vulvar SCC (20). A higher number of RLNs may increase the potential for lymph node micrometastases. However, in a study that included 158 vulvar SCC patients who underwent bilateral inguinofemoral lymphadenectomy, Baiocchi et al. found that a higher RLN count did not correlate with lymph node involvement (14). In our study, we observed a significant difference in the PLN count according to various RLN count groups, with a significantly higher number of PLNs found in patients with a higher number of RLNs. Based on our results, we confirmed our hypothesis that a higher RLN count may more accurately predict nodal status in vulvar SCC patients with node-positive disease.

Our results also found that older age was associated with fewer RLNs. The reason for this difference remains unclear. Since all of patients identified in the study underwent surgery and lymphadenectomy, we can presume that these patients were not subject to serious complications and were suitable for surgical treatment and lymphadenectomy. A study by Panici et al. showed that the survival benefit of lymphadenectomy in older patients was similar to that of younger patients, and that lymphadenectomy did not increase the probability of complications (21). Although we could not obtain surgical complication data from the SEER database, several previous studies have confirmed that lymphadenectomy does not have higher complication rates such as wound breakdown and lymphedema in older patients than in younger patients (21-24). These data suggest that complete lymphadenectomy is an important intervention in older patients too.

The optimal number of lymph nodes that should be removed during inguinal lymphadenectomy remains unclear. Baiocchi et al. found that in the $50.6 \%$ of study patients with node-positive disease, there was no significant difference in outcomes between patients with $<12$ RLNs and those with $\geq 12$, while resection of $<12$ lymph nodes in node-positive disease negatively affected survival outcomes $(p<0.05)(14)$. However, a study by Gill et al. did not find any difference in survival according to number of PLNs in univariate analysis. In our population-based study, we observed that resection of $\leq 6$ lymph nodes had a significantly negative impact on CSS and OS (13).

Adjuvant radiotherapy has been confirmed to improve outcomes in patients with node-positive vulvar SCC in previous research (16-19). A total of $69.7 \%$ of the patients in our study underwent adjuvant radiotherapy; however, adjuvant radiotherapy had no effect on survival outcomes of patients, according to the results of multivariate analyses. Very few studies have assessed the value of adjuvant radiotherapy according to the number of RLNs. Parthasarathy et al. studied 208 patients with a single PLN, and their results suggested that adjuvant radiotherapy may improve the disease-specific survival of single PLN patients with $\leq 12$ RLNs (18). A study by Polterauer et al. found that adjuvant radiotherapy only improved survival in patients with a lymph node ratio $>20 \%$, and not in patients with a lymph node ratio $\leq 20 \%$ (25). In our series, adjuvant radiotherapy has been statistically shown to improve outcomes in patients who received a less extensive lymphadenectomy ( $\leq 6 \mathrm{RLNs})$, while not affecting the survival outcomes of patients with $\geq 7$ RLNs. Therefore, assessment of the number of RLNs may not only indicate the potential therapeutic effect of lymphadenectomy, but may also be a useful method of selecting appropriate candidates for adjuvant radiotherapy.

Several limitations of our study should be acknowledged. First, the SEER database lacks a centralized pathology review, causing potential variability and subjectivity in determining the number of RLNs, and patterns of disease recurrence were also not recorded. Second, tumor location, such as midline, whether bilateral groin lymphadenectomy was performed, and the RLN count for each side or per patient was also not available in the SEER program. Third, details of the lymphadenectomy techniques used-debulking of bulky lymph nodes, superficial groin dissection, or superficial plus deep groin dissection-were lacking. Moreover, the cutoff point of the number of RLNs was classified based on quartiles. The optimal cutoff point of the number of RLNs should be explored in future prospective multicenter studies. Many of these limitations could be overcome by evaluating patients attending a single institution, but the rarity of the disease means that the use of a population-based database such as the SEER program, with its inherent strengths and weaknesses, is required.

\section{CONCLUSION}

In conclusion, our results suggest that RLN count is an independent predictor of outcome in vulvar SCC with node-positive disease. Patients with $>6$ RLNs had a significantly higher CSS and OS than patients with $\leq 6$ RLNs. Further prospective studies with more patients are needed to further evaluate the role of removal of lymph nodes in vulvar SCC.

\section{ETHICS STATEMENT}

The institutional review board of the First Affiliated Hospital of Xiamen University had approved this study.

\section{AUTHOR CONTRIBUTIONS}

S-GW, W-WZ, Z-YH, and JZ are lead authors who participated in data collection, manuscript drafting, table/figure creation, and manuscript revision. W-WZ, Q-HC, and J-YS aided in data collection. S-GW is the dosimetrist who contributed dosimetric data and figures. S-GW and JZ are senior authors who aided in drafing the manuscript and manuscript revision. Z-YH and JZ are corresponding authors who initially developed the concept and drafted and revised the manuscript. All authors read and approved the final manuscript. 


\section{FUNDING}

This work was supported by grants from the Natural Science Foundation of Fujian Province (No. 2015J01550,

\section{REFERENCES}

1. Siegel R, Naishadham D, Jemal A. Cancer statistics, 2013. CA Cancer J Clin (2013) 63(1):11-30. doi:10.3322/caac.21166

2. Hacker NF, Eifel PJ, van der Velden J. Cancer of the vulva. Int J Gynaecol Obstet (2012) 119(Suppl 2):S90-6. doi:10.1016/S0020-7292(12)60022-8

3. Zhou J, Shan G. The prognostic role of FIGO stage in patients with vulvar cancer: a systematic review and meta-analysis. Curr Med Res Opin (2016) 32(6):1121-30. doi:10.1185/03007995.2016.1162147

4. Le T, Elsugi R, Hopkins L, Faught W, Fung-Kee-Fung M. The definition of optimal inguinal femoral nodal dissection in the management of vulva squamous cell carcinoma. Ann Surg Oncol (2007) 14(7):2128-32. doi:10.1245/ s10434-007-9417-5

5. Stehman FB, Ali S, DiSaia PJ. Node count and groin recurrence in early vulvar cancer: a Gynecologic Oncology Group study. Gynecol Oncol (2009) 113(1):52-6. doi:10.1016/j.ygyno.2008.12.028

6. Courtney-Brooks M, Sukumvanich P, Beriwal S, Zorn KK, Richard SD, Krivak TC. Does the number of nodes removed impact survival in vulvar cancer patients with node-negative disease? Gynecol Oncol (2010) 117(2):308-11. doi:10.1016/j.ygyno.2010.01.007

7. Gordinier ME, Malpica A, Burke TW, Bodurka DC, Wolf JK, Jhingran A, et al. Groin recurrence in patients with vulvar cancer with negative nodes on superficial inguinal lymphadenectomy. Gynecol Oncol (2003) 90(3):625-8. doi:10.1016/S0090-8258(03)00374-3

8. Gadducci A, Ferrero A, Tana R, Fabrini MG, Modaffari P, Fanucchi A, et al. Prognostic value of lymph node status and number of removed nodes in patients with squamous cell carcinoma of the vulva treated with modified radical vulvectomy and inguinal-femoral lymphadenectomy. Eur J Gynaecol Oncol (2012) 33(6):640-3.

9. Van der Zee AG, Oonk MH, De Hullu JA, Ansink AC, Vergote I, Verheijen $\mathrm{RH}$, et al. Sentinel node dissection is safe in the treatment of early-stage vulvar cancer. J Clin Oncol (2008) 26(6):884-9. doi:10.1200/JCO.2007.14.0566

10. Levenback CF, Ali S, Coleman RL, Gold MA, Fowler JM, Judson PL, et al. Lymphatic mapping and sentinel lymph node biopsy in women with squamous cell carcinoma of the vulva: a gynecologic oncology group study. J Clin Oncol (2012) 30(31):3786-91. doi:10.1200/JCO.2011.41.2528

11. Te Grootenhuis NC, van der Zee AG, van Doorn HC, van der Velden J, Vergote I, Zanagnolo V, et al. Sentinel nodes in vulvar cancer: long-term follow-up of the GROningen INternational Study on sentinel nodes in vulvar cancer (GROINSS-V) I. Gynecol Oncol (2016) 140(1):8-14. doi:10.1016/j. ygyno.2015.09.077

12. Zhou J, Zhang WW, Chen XT, Wu SG, Sun JY, Chen QH, et al. The trends and outcomes of sentinel lymph node biopsy in early stage vulvar squamous cell carcinoma: a population-based study. JCancer (2018) 9(11):1951-7. doi:10.7150/jca.24044

13. Gill BS, Bernard ME, Lin JF, Balasubramani GK, Rajagopalan MS, Sukumvanich $\mathrm{P}$, et al. Impact of adjuvant chemotherapy with radiation for node-positive vulvar cancer: a National Cancer Data Base (NCDB) analysis. Gynecol Oncol (2015) 137(3):365-72. doi:10.1016/j.ygyno.2015.03.056

14. Baiocchi G, Cestari FM, Rocha RM, Faloppa CC, Kumagai LY, Fukazawa EM, et al. Does the count after inguinofemoral lymphadenectomy in vulvar cancer correlate with outcome? Eur J Surg Oncol (2013) 39(4):339-43. doi:10.1016/j. ejso.2013.02.012

15. Surveillance, Epidemiology, and End Results (SEER) Program (www.seer.cancer.gov) SEER ${ }^{\star}$ Stat Database: Incidence - SEER 18 Regs Custom Data (with
2013D001), the Medical Innovation Foundation of Fujian Province (No. 2015-CXB-34), and the Foundation for Young Scholar of Fujian Provincial Health Department (No. 2014ZQN-ZD-31).

chemotherapy recode), Nov 2015 Sub (2000-2013) <Katrina/Rita Population Adjustment > - Linked To County Attributes - Total U.S., 1969-2014 Counties, National Cancer Institute, DCCPS, Surveillance Research Program, released July 2016, based on the November 2015 submission.

16. Homesley HD, Bundy BN, Sedlis A, Adcock L. Radiation therapy versus pelvic node resection for carcinoma of the vulva with positive groin nodes. Obstet Gynecol (1986) 68(6):733-40.

17. Mahner S, Jueckstock J, Hilpert F, Neuser P, Harter P, de Gregorio N, et al. Adjuvant therapy in lymph node-positive vulvar cancer: the AGO-CaRE-1 study. J Natl Cancer Inst (2015) 107(3):dju426. doi:10.1093/jnci/dju426

18. Parthasarathy A, Cheung MK, Osann K, Husain A, Teng NN, Berek JS, et al. The benefit of adjuvant radiation therapy in single-node-positive squamous cell vulvar carcinoma. Gynecol Oncol (2006) 103(3):1095-9. doi:10.1016/j. ygyno.2006.06.030

19. Swanick CW, Eifel PJ, Huo J, Meyer LA, Smith GL. Challenges to delivery and effectiveness of adjuvant radiation therapy in elderly patients with nodepositive vulvar cancer. Gynecol Oncol (2017) 146(1):87-93. doi:10.1016/j. ygyno.2017.05.004

20. Pecorelli S. Revised FIGO staging for carcinoma of the vulva, cervix, and endometrium. Int J Gynaecol Obstet (2009) 105(2):103-4. doi:10.1016/j. ijgo.2009.02.009

21. Panici PB, Tomao F, Domenici L, Giannini A, Giannarelli D, Palaia I, et al Prognostic role of inguinal lymphadenectomy in vulvar squamous carcinoma: younger and older patients should be equally treated. A prospective study and literature review. Gynecol Oncol (2015) 137(3):373-9. doi:10.1016/j. ygyno.2015.03.013

22. Walker KF, Day H, Abu J, Nunns D, Williamson K, Duncan T. Do surgical techniques used in groin lymphadenectomy for vulval cancer affect morbidity rates? Int J Gynecol Cancer (2011) 21(8):1495-9. doi:10.1097/ IGC.0b013e318228f314

23. Hinten F, van den Einden LC, Hendriks JC, van der Zee AG, Bulten J, Massuger LF, et al. Risk factors for short- and long-term complications after groin surgery in vulvar cancer. Br J Cancer (2011) 105(9):1279-87. doi:10.1038/bjc.2011.407

24. Gaarenstroom KN, Kenter GG, Trimbos JB, Agous I, Amant F, Peters AA, et al. Postoperative complications after vulvectomy and inguinofemoral lymphadenectomy using separate groin incisions. Int J Gynecol Cancer (2003) 13(4):522-7. doi:10.1046/j.1525-1438.2003.13304.x

25. Polterauer S, Schwameis R, Grimm C, Macuks R, Iacoponi S, Zalewski K, et al. Prognostic value of lymph node ratio and number of positive inguinal nodes in patients with vulvar cancer. Gynecol Oncol (2017) 147(1):92-7. doi:10.1016/j.ygyno.2017.07.142

Conflict of Interest Statement: The authors declare that the research was conducted in the absence of any commercial or financial relationships that could be construed as a potential conflict of interest.

The reviewer MS and handling Editor declared their shared affiliation.

Copyright (c) $2018 \mathrm{Wu}$, Zhang, Sun, Chen, He and Zhou. This is an open-access article distributed under the terms of the Creative Commons Attribution License (CC BY). The use, distribution or reproduction in other forums is permitted, provided the original author(s) and the copyright owner are credited and that the original publication in this journal is cited, in accordance with accepted academic practice. No use, distribution or reproduction is permitted which does not comply with these terms. 